\title{
Model for the Interaction of Thermal Radiation with a Monatomic Plasma
}

\author{
J. R. Saraf \\ Mathematics, School of Physical Sciences, The New University of Ulster, Coleraine, \\ Northern Ireland, U.K.
}

(Z. Naturforsch. 31 a, 1038-1041 [1976] ; received May 19, 1976)

\begin{abstract}
A kinetic model for a weakly-ionized plasma interacting with radiation is proposed based on the energy-momentum method, in which both energy and momentum of the system are conserved. The radiation-initiated ionization is obtained in a non-inertial coordinate system, and the proposed model satisfies the H-theorem.
\end{abstract}

\section{Introduction}

The advent of laser technology and possibility of laser-driven thermonuclear fusion ${ }^{1}$ has generated interest in the problem of a plasma interacting with thermal radiation. The laser radiation plays quite a varied role, and radiative forces have been known to contribute to phenomena such as plasma acceleration $^{2}$, self-focusing of laser radiation ${ }^{3}$, magnetic field generation ${ }^{4}$, and parametric instabilities ${ }^{5}$.

Recently, kinetic models for the interaction of thermal radiation with a rarefied diatomic gas have been proposed by Phillips and Arpaci ${ }^{6}$ and Saraf ${ }^{7-9}$. Though the coupling of radiation field with an ionized gas has been widely investigated from the continuum viewpoint ${ }^{\mathbf{1 0}}$, there have recently been two attempts by Anderson, Baum and Krook ${ }^{11}$, and by Phillips and Arpaci ${ }^{12}$ to study this coupling at the level of kinetic theory ${ }^{13}$. The energy method ${ }^{6,7}$ used by Phillips and Arpaci ${ }^{12}$ conserves the total energy of isolated system of plasma and radiation but completely ignores the contributions of the particle and radiative momentums. However, the exchange of momentum between the radiation field and plasma is important in laser-induced fusion wherein intense optical radiation is absorbed by the plasma. In addition, Phillips and Arpaci ${ }^{12}$ have neglected the effect of macroscopic motion of plasma on radiation so that the radiative transfer equation applies only to an inertial coordinate system. The Boltzmann equations for material particles are conveniently written in a non-inertial frame and moreover, in such a frame the absorption coefficient of radiation is usually independent of the direction of

Reprint requests to Dr. J. R. Saraf, Dept. of Mathematics, School of Physical Sciences, The New University of Ulster, Coleraine, Northern Ireland, U.K. propagation of photons. Hence, Saraf ${ }^{7,8}$ used radiative transport equations ${ }^{13}$ in a non-inertial system to formulate models for the interaction of radiation with a diatomic gas.

In this paper, we use the energy-momentum $\operatorname{method}^{8}$ to model a weakly ionized plasma interacting with radiation. The radiative transfer equaquation in non-inertial frame is employed for obtaining model equations for photon-initiated ionization.

\section{Formulation}

The analysis in this study is based on all the assumptions listed in $\S 2$ of Phillips and Arpaci ${ }^{12}$, with the exception of $(\mathrm{V})$. We consider a weaklyionized three-component plasma comprised of electrons, ions and atoms which are assumed to be monatomic particles. A non-equilibrium degree of ionization is defined as

$$
\Phi=\left(n_{\mathrm{i}} n_{\mathrm{e}} / n^{2}\right)^{1 / 2},
$$

where $n_{\mathrm{i}}, n_{\mathrm{e}}$ and $n$ denote, respectively, the number density of ions, electrons and heavy particles (atoms plus ions). In equilibrium, $n_{\mathrm{i}}=n_{\mathrm{e}}=n_{\mathrm{e} 0}$ and the equilibrium degree of ionization $\Phi_{0}=n_{\mathrm{e} 0} / n$ in the special case of weak $\left(\Phi, \Phi_{0} \ll 1\right)$ one-fold ionization can be shown ${ }^{12}$ to be

$$
\Phi_{0} \cong\left(\frac{T}{\Theta_{\mathrm{e}}}\right)^{5 / 4}\left(\frac{p_{0}}{p}\right)^{1 / 2}\left(\frac{Z_{\mathrm{e}}^{\mathrm{i}}}{Z_{\mathrm{e}}^{\mathrm{a}}}\right)^{1 / 2} \exp \left\{-\Theta_{\mathrm{I}} / 2 T\right\},
$$

where $p$ is the pressure, $T$ is the temperature, $\Theta_{\mathrm{e}}$ is electronic temperature, $p_{0}$ is the standard atmospheric pressure, $\Theta_{\mathrm{I}}\left(=\varepsilon_{\mathrm{I}} / k\right)$ is the characteristic temperature for ionization, $k$ is Boltzmann constant, $\varepsilon_{\mathrm{I}}$ is the ionization potential, and $Z_{\mathrm{e}}{ }^{\mathrm{i}}$ and $Z_{\mathrm{e}}{ }^{\mathrm{a}}$ are respectively, the electronic partition functions for the ion and atom. 
Assuming ideal gas law $p=n\left(1+\Phi_{0}\right) k T$, we have

$$
\left(\frac{\partial \Phi_{0}}{\partial T}\right)_{\mathrm{n}}=\frac{1}{2}\left(\frac{3}{2}+\frac{\Theta_{\mathrm{I}}}{T}\right) \frac{\Phi_{0}}{T} .
$$

In terms of internal energy $u_{0}=\frac{3}{2}\left(1+\Phi_{0}\right) R_{\mathrm{a}} T$ $+\Phi_{0} R_{\mathrm{a}} \Theta_{\mathrm{I}}$, the specific heat at constant volume is $C_{\mathrm{v}}=\left(\frac{\partial u_{0}}{\partial T}\right)_{\mathrm{n}}=\frac{3}{2}\left(1+\Phi_{0}\right) R_{\mathrm{a}}+\frac{1}{2}\left(\frac{3}{2}+\frac{\Theta_{\mathrm{I}}}{T}\right)^{2} \Phi_{0} R_{\mathrm{a}}$,

where $R_{\mathrm{a}}$ is gas constant for the atom.

Denoting by $f^{r}(\boldsymbol{x}, \boldsymbol{V}, t)$ the velocity distribution functions, where $\boldsymbol{V}$ is particle velocity, the mean velocity $\langle\boldsymbol{V}\rangle_{r}$ and temperature $T_{r}$ of the $r$-species are defined as

$$
n_{r}\langle\boldsymbol{V}\rangle_{r}=\int n_{r} f^{r} \mathrm{~d} \boldsymbol{V} \mathrm{d} \Omega,
$$

and

$$
3 k n_{r} T_{r}=\int m_{r} \boldsymbol{U}_{r}^{2} n_{r} f^{r} \mathrm{~d} \boldsymbol{V} \mathrm{d} \Omega \equiv m_{r} n_{r}\left\langle\mathbf{U}_{r}^{2}\right\rangle_{r},
$$

where

$$
\boldsymbol{U}_{r}=\boldsymbol{V}-\langle\boldsymbol{V}\rangle_{r},
$$

is the random thermal velocity of the $r$-species, and $m_{r}$ is mass of an $r$-particle.

The mean mass, or drift velocity of the plasma is defined as

$$
\overline{\mathbf{V}}=\sum_{r} \boldsymbol{m}_{r} \boldsymbol{n}_{r}\langle\mathbf{V}\rangle_{r} / \sum_{r} \boldsymbol{m}_{r} \boldsymbol{n}_{r} .
$$

The thermal velocity is defined as

$$
\mathbf{U}=\mathbf{V}-\overline{\mathbf{V}} \text {. }
$$

The model kinetic equations for plasma-radiation interaction proposed by Phillips and Arpaci ${ }^{12}$ are

$$
\begin{aligned}
& \frac{\partial}{\partial t}\left(n_{r} f^{r}\right)+\boldsymbol{V} \cdot \frac{\partial}{\partial \boldsymbol{x}}\left(n_{r} f^{r}\right)+\frac{\boldsymbol{F}^{r}}{m_{r}} \cdot \frac{\partial}{\partial \boldsymbol{V}}\left(n_{r} f^{r}\right) \\
& =\sum_{s}\left[\frac{\delta}{\delta t}\left(n_{r} f^{r}\right)\right]_{\mathrm{S}}+\left[\frac{\delta}{\delta t}\left(n_{r} f^{r}\right)\right]_{\mathrm{M}}^{\mathrm{I}}+\left[\frac{\delta}{\delta t}\left(n_{r} f^{r}\right)\right]_{\mathrm{P}}^{\mathrm{I}},
\end{aligned}
$$

where $\boldsymbol{F}^{r}$ is applied force, and the indices $r, s$ apply to electrons, ions and atoms.

\section{A) Elastic Collisions}

The summation term on rhs of (10) represents the elastic self- and cross-collisions between the electrons, ions, and atoms, and could be written as

$$
\sum_{s}\left[\frac{\delta}{\delta t}\left(n_{r} f^{r}\right)\right]_{\mathrm{s}}=\sum_{s} n_{r} n_{s} \sigma_{r s}\langle\boldsymbol{U}\rangle_{r s}\left(f_{0 s}^{r}-f^{r}\right),
$$

where $\sigma_{r s}$ is the collision cross-section, and

$$
f_{0 s}^{r}=\left(\frac{m_{r}}{2 \pi k T_{r s}}\right)^{3 / 2} \exp \left[-\frac{m_{r}}{2 k T_{r s}}\left(\boldsymbol{V}-\langle\boldsymbol{V}\rangle_{r s}\right)^{2}\right],
$$

and $\quad\langle\boldsymbol{U}\rangle_{r s}=\left[\left(\langle\boldsymbol{U}\rangle_{r}\right)^{2}+\left(\langle\boldsymbol{U}\rangle_{s}\right)^{2}\right]^{1 / 2}$.

The quantities $\langle\boldsymbol{V}\rangle_{r s}$ and $T_{r s}$ are well-known to be

$$
\langle\boldsymbol{V}\rangle_{r s}=\frac{m_{r}\langle\mathbf{V}\rangle_{r}+m_{s}\langle\mathbf{V}\rangle_{s}}{m_{r}+m_{s}},
$$

and

$$
\begin{aligned}
& T_{r s}=\frac{1}{6 k}\left[m_{r}\left\langle\boldsymbol{U}_{r}{ }^{2}\right\rangle_{r}+m_{s}\left\langle\boldsymbol{U}_{s}{ }^{2}\right\rangle_{s}\right. \\
&\left.+\frac{m_{r} m_{s}}{m_{r}+m_{s}}\left(\langle\boldsymbol{V}\rangle_{r}-\langle\boldsymbol{V}\rangle_{s}\right)^{2}\right] .
\end{aligned}
$$

We note that for $r=s,\langle\boldsymbol{V}\rangle_{r r}=\langle\boldsymbol{V}\rangle_{r}, T_{r r}=T_{r}$ so that $f_{0 r}^{r}$ is the Maxwellian distribution for $r$-species at temperature $T_{r}$ and mean velocity $\langle\boldsymbol{V}\rangle_{r}$, given by

$$
f_{0 r}^{r}=\left(\frac{m_{r}}{2 \pi k T_{r}}\right)^{3 / 2} \exp \left\{-m_{r} \boldsymbol{U}_{r}^{2} / 2 k T_{r}\right\}
$$

where $\boldsymbol{U}_{r}$ is defined by (7).

\section{B) Particle Ionization}

Phillips and Arpaci ${ }^{12}$ noted that the depleted ions and electrons are associated with the replenished atoms, and vice versa. The particle-particle ionization represented by second term on rhs of (10) can be modelled as ${ }^{12}$

$$
\begin{aligned}
& {\left[\frac{\delta}{\delta t}\left(n_{\mathrm{a}} f^{\mathrm{a}}\right)\right]_{\mathrm{M}}^{\mathrm{I}}=n^{2} \sigma_{\mathrm{M}}^{\mathrm{I}}\langle\mathbf{U}\rangle_{\mathrm{ei}}\left(\Phi^{2} f_{0}^{\mathrm{a}}-\Phi_{0}{ }^{2} f^{\mathrm{a}}\right),} \\
& {\left[\frac{\delta}{\delta t}\left(n_{\mathrm{i}} f^{\mathrm{i}}\right)\right]_{\mathrm{M}}^{\mathrm{I}}=n^{2} \sigma_{\mathrm{M}}^{\mathrm{I}}\langle\mathbf{U}\rangle_{\mathrm{ei}}\left(\Phi_{0}{ }^{2} f_{0}^{\mathrm{i}}-\Phi^{2} f^{\mathrm{i}}\right),} \\
& \text { and } \\
& {\left[\frac{\delta}{\delta t}\left(n_{\mathrm{e}} f^{\mathrm{e}}\right)\right]_{\mathrm{M}}^{\mathrm{I}}=n^{2} \sigma_{\mathrm{M}}^{\mathrm{I}}\langle\mathbf{U}\rangle_{\mathrm{ei}}\left(\Phi_{0}{ }^{2} f_{0}^{\mathrm{e}}-\Phi^{2} f^{\mathrm{e}}\right),}
\end{aligned}
$$

where $\sigma_{\mathrm{M}}^{\mathrm{I}}$ denotes the collision cross-section for the particle-initiated ionization, and

$$
\begin{gathered}
f_{0}{ }^{r}=\left(\frac{m_{r}}{2 \pi k T_{\mathrm{M}}^{\mathrm{I}}}\right)^{3 / 2} \exp \left[-m_{r}\left(\boldsymbol{V}-\langle\mathbf{V}\rangle_{\mathrm{M}}^{\mathrm{I}}\right)^{2} /\left(2 k T_{\mathrm{M}}^{\mathrm{I}}\right)\right] \\
{\left[1+\left(\frac{\Phi}{\Phi_{0}}\right)^{2}\right]\langle\boldsymbol{V}\rangle_{\mathrm{M}}^{\mathrm{I}}=\langle\mathbf{V}\rangle_{\mathrm{a}}} \\
\quad+\left(\frac{\Phi}{\Phi_{0}}\right)^{2}\left[\frac{m_{\mathrm{e}}}{m_{\mathrm{a}}}\langle\boldsymbol{V}\rangle_{\mathrm{e}}+\frac{m_{\mathrm{i}}}{m_{\mathrm{a}}}\langle\boldsymbol{V}\rangle_{\mathrm{i}}\right],
\end{gathered}
$$

and

$$
\begin{aligned}
{\left[1+\left(\frac{\Phi}{\Phi_{0}}\right)^{2}\right] T_{\mathrm{M}}^{\mathrm{I}} \cong T_{\mathrm{a}} } & +\left(\frac{\Phi}{\Phi_{0}}\right)^{2}\left(T_{\mathrm{e}}+T_{\mathrm{i}}\right) \\
& +\frac{2}{3} \Theta_{\mathrm{I}}\left[\left(\frac{\Phi}{\Phi_{0}}\right)^{2}-1\right] .
\end{aligned}
$$




\section{C) Radiative Transfer}

The radiation is taken to have a continuous spectrum in visible or ultra-violet region, resulting from bound-free transitions. Since heavy particles are assumed to remain in their electronic ground state, the line radiation from bound-bound transitions is absent. Also, we neglect the photon-photon scattering as well as the continuous radiation related to electron-photon interaction. The evolution of specific intensity $I_{v}$ of radiation is given by the radiative transfer equation

$$
\frac{1}{c} \frac{\partial I_{v}}{\partial t}+\boldsymbol{l} \cdot \frac{\partial I_{v}}{\partial \boldsymbol{x}}=K_{v}\left(I_{v 0}-I_{v}\right)
$$

where $c$ is speed of light, $v$ is spectral frequency, $\boldsymbol{l}$ is the propagation direction, $K_{v}$ is the coefficient of monochromatic absorption, and $I_{\nu 0}$ is the equilibrium Planck distribution at "local" temperature $T_{\mathrm{e}}$ for electron-species:

$$
I_{v 0}=\frac{2 h v^{3}}{c^{2}}\left[\exp \left\{h v / k T_{\mathrm{e}}\right\}-1\right]^{-1},
$$

where $h$ is Planck's constant.

Finally, we note that the last term on rhs of (10) represents radiation-initiated ionization and we now turn to model this term.

\section{Photon Ionization by Energy-Momentum Method $^{8}$}

As an electron is very much lighter than an ion or an atom, the photons mainly collide with electrons in the plasma, and thus the radiative interactions do not appreciably change the momentum and kinetic energy of the heavy particles. Sampson ${ }^{13}$ has obtained transport equations for radiative momentum and energy in non-inertial frames of reference. Now, according to the energy-momentum principle $^{8}$, the radiative momentum is equated to the rate of change in momentum of electrons, while the radiative energy is balanced by the rate of change in the energy of electrons and with the ionization of atoms. Thus, we have

$\left[\frac{\delta}{\delta t}\left(m_{\mathrm{e}} n_{\mathrm{e}}\langle\boldsymbol{V}\rangle_{\mathrm{e}}\right)\right]_{\mathrm{P}}^{\mathrm{I}}=\frac{1}{c} \int K_{\nu} \boldsymbol{l}\left(I_{\nu}-I_{\nu 0}\right) \mathrm{d} v \mathrm{~d} \Omega$,

and

$$
\begin{aligned}
{\left[\frac { \delta } { \delta t } \left(\frac{1}{2} m_{\mathrm{e}}\right.\right.} & \left.\left.n_{\mathrm{e}}\left\langle\boldsymbol{U}_{\mathrm{e}}^{2}\right\rangle_{\mathrm{e}}\right)\right]_{\mathrm{P}}^{\mathrm{I}}+\varepsilon_{\mathrm{I}}\left[\frac{\delta n_{\mathrm{i}}}{\delta t}\right]_{\mathrm{P}}^{\mathrm{I}} \\
& =\int K_{v}\left(I_{v}-I_{v 0}-\frac{1}{c} \overline{\boldsymbol{V}} \cdot \boldsymbol{l} I_{v}\right) \mathrm{d} v \mathrm{~d} \Omega .
\end{aligned}
$$

We can rearrange the last term in (10) as

$$
\begin{aligned}
& {\left[\frac{\delta}{\delta t}\left(n_{\mathrm{e}} f^{\mathrm{e}}\right)\right]_{\mathrm{P}}^{\mathrm{I}}=\frac{\partial\left(n_{\mathrm{e}} f^{\mathrm{e}}\right)}{\partial\left(m_{\mathrm{e}} n_{\mathrm{e}}\langle\boldsymbol{V}\rangle_{\mathrm{e}}\right)}\left[\frac{\delta}{\delta t}\left(m_{\mathrm{e}} n_{\mathrm{e}}\langle\boldsymbol{V}\rangle_{\mathrm{e}}\right)\right]_{\mathrm{P}}^{\mathrm{I}}} \\
& +\frac{\partial\left(n_{\mathrm{e}} f^{\mathrm{e}}\right)}{\partial\left(\frac{1}{2} m_{\mathrm{e}} n_{\mathrm{e}}\left\langle\mathbf{U}_{\mathrm{e}}{ }^{2}\right\rangle_{\mathrm{e}}\right)}\left[\frac{\delta}{\delta t}\left(\frac{1}{2} m_{\mathrm{e}} n_{\mathrm{e}}\left\langle\boldsymbol{U}_{\mathrm{e}}{ }^{2}\right\rangle_{\mathrm{e}}\right)\right]_{\mathrm{P}}^{\mathrm{I}} .(27)
\end{aligned}
$$

As the mean free path of a photon is much larger than that of an electron, we can take electrons to be distributed according to Maxwellian equilibrium $f_{0 \mathrm{e}}^{\mathrm{e}}$ [given by (16)] during photon relaxation, and write

$$
\frac{\partial\left(n_{\mathrm{e}} f^{\mathrm{e}}\right)}{\partial\left(m_{\mathrm{e}} n_{\mathrm{e}}\langle\boldsymbol{V}\rangle_{\mathrm{e}}\right)}=\frac{\partial\left(n_{\mathrm{e} 0} f_{0 \mathrm{e}}^{\mathrm{e}}\right)}{\partial\left(m_{\mathrm{e}} n_{\mathrm{e} 0}\langle\boldsymbol{V}\rangle_{\mathrm{e}}\right)}
$$

and

$$
\frac{\partial\left(n_{\mathrm{e}} f^{\mathrm{e}}\right)}{\partial\left(\frac{1}{2} m_{\mathrm{e}} n_{\mathrm{e}}\left\langle\mathbf{U}_{\mathrm{e}}^{2}\right\rangle_{\mathrm{e}}\right)}=\frac{\partial\left(n_{\mathrm{e} 0} f_{\mathrm{e}}^{\mathrm{e}}\right) / \partial T_{\mathrm{e}}}{\partial\left(\frac{1}{2} m_{\mathrm{e}} n_{\mathrm{e} 0}\left\langle\mathbf{U}_{\mathrm{e}}^{2}\right\rangle_{\mathrm{e}}\right) / \partial T_{\mathrm{e}}} .
$$

But, from (6), (3) and (16), we have

$$
\begin{gathered}
m_{\mathrm{e}}\left\langle\boldsymbol{U}_{\mathrm{e}}{ }^{2}\right\rangle_{\mathrm{e}}=3 k T_{\mathrm{e}}, \\
\frac{\partial n_{\mathrm{e} 0}}{\partial T_{\mathrm{e}}}=\frac{1}{2}\left(\frac{3}{2}+\frac{\Theta_{\mathrm{I}}}{T_{\mathrm{e}}}\right) \frac{n_{\mathrm{e} 0}}{T_{\mathrm{e}}},
\end{gathered}
$$

and

$$
\frac{\partial f_{0 \mathrm{e}}^{\mathrm{e}}}{\partial T_{\mathrm{e}}}=\left(\frac{m_{\mathrm{e}} \boldsymbol{U}_{\mathrm{e}}^{2}}{2 k T_{\mathrm{e}}}-\frac{3}{2}\right) \frac{f_{0 \mathrm{e}}^{\mathrm{e}}}{T_{\mathrm{e}}} .
$$

Thus, we can rewrite (27) as

$$
\begin{aligned}
{\left[\frac{\delta}{\delta t}\left(n_{\mathrm{e}} f^{\mathrm{e}}\right)\right]_{\mathrm{P}}^{\mathrm{I}}=} & \frac{\boldsymbol{U}_{\mathrm{e}} f_{0 \mathrm{e}}^{\mathrm{e}}}{c k T_{\mathrm{e}}} \cdot \int K_{v} \boldsymbol{l}\left(I_{v}-I_{v 0}\right) \mathrm{d} v \mathrm{~d} \Omega+\frac{\left(3 k T_{\mathrm{e}}-2 \varepsilon_{\mathrm{I}}-2 m_{\mathrm{e}} \boldsymbol{U}_{\mathrm{e}}^{2}\right) f_{0 \mathrm{e}}^{\mathrm{e}}}{3 k T_{\mathrm{e}}\left(\varepsilon_{\mathrm{I}}+7 k T_{\mathrm{e}} / 2\right)}\left\{\varepsilon_{\mathrm{I}}\left[\frac{\delta n_{\mathrm{i}}}{\delta t}\right]_{\mathrm{P}}^{\mathrm{I}}\right. \\
& \left.+\int K_{v}\left(I_{v 0}-I_{v}-\frac{1}{c} \overline{\boldsymbol{V}} \cdot \boldsymbol{l} l_{v}\right) \mathrm{d} v \mathrm{~d} \Omega\right\} .
\end{aligned}
$$

Since charge is conserved, we have

$$
\begin{aligned}
& {\left[\frac{\delta n_{\mathrm{i}}}{\delta t}\right]_{\mathrm{P}}^{\mathrm{I}}=\left[\frac{\delta n_{\mathrm{e}}}{\delta t}\right]_{\mathrm{P}}^{\mathrm{I}}=\int\left[\frac{\delta}{\delta t}\left(n_{\mathrm{e}} f^{\mathrm{e}}\right)\right]_{\mathrm{P}}^{\mathrm{I}} \mathrm{d} \Omega \mathrm{d} \boldsymbol{V}=\frac{\left(3 k T_{\mathrm{e}}+2 \varepsilon_{\mathrm{I}}\right) R_{\mathrm{a}} \Phi_{0}}{4\left(k T_{\mathrm{e}}\right)^{2} C_{\mathrm{v}}{ }^{\mathrm{I}}} \int K_{\nu}\left(I_{v}-I_{r 0}+\frac{1}{c} \overline{\boldsymbol{V}} \cdot \boldsymbol{l} I_{v}\right) \mathrm{d} \nu \mathrm{d} \Omega,} \\
& \text { where } \\
& C_{\mathrm{v}}{ }^{\mathrm{I}}=C_{\mathrm{v}}-3 R_{\mathrm{a}} / 2 .
\end{aligned}
$$


And, as the ionization does not change the number density of heavy particles, we have

$$
\left[\frac{\delta n_{\mathrm{a}}}{\delta t}\right]_{\mathrm{P}}^{\mathrm{I}}+\left[\frac{\delta n_{\mathrm{i}}}{\delta t}\right]_{\mathrm{P}}^{\mathrm{I}}=0 \text {. }
$$

As noted at the beginning of this section, photons do not affect the momentum and energy of ions and atoms but can only change their number densities, we obtain from (34) and (35) the collision terms for ions and atoms due to photon-initiated ionization, as

$$
\left[\frac{\delta\left(n_{\mathrm{i}} f^{\mathrm{i}}\right)}{\delta t}\right]_{\mathrm{P}}^{\mathrm{I}}=\frac{\left(3 k T_{\mathrm{e}}+2 \varepsilon_{\mathrm{I}}\right) R_{\mathrm{a}} \Phi_{0} f_{0 \mathrm{i}}^{\mathrm{i}}}{4\left(k T_{\mathrm{e}}\right)^{2} C_{\mathrm{v}}^{\mathrm{I}}} \int K_{\nu}\left(I_{v}-I_{v 0}+\frac{1}{c} \overline{\mathbf{V}} \cdot \boldsymbol{l} I_{v}\right) \mathrm{d} v \mathrm{~d} \Omega,
$$

and

$$
\left[\frac{\delta\left(n_{\mathrm{a}} f^{\mathrm{a}}\right)}{\delta t}\right]_{\mathrm{P}}^{\mathrm{I}}=\frac{\left(3 k T_{\mathrm{e}}+2 \varepsilon_{\mathrm{I}}\right) R_{\mathrm{a}} \Phi_{0} f_{0 \mathrm{a}}^{\mathrm{a}}}{4\left(k T_{\mathrm{e}}\right)^{2} C_{\mathrm{v}}{ }^{\mathrm{I}}} \int K_{\nu}\left(I_{\nu 0}-I_{v}-\frac{1}{c} \overline{\mathbf{V}} \cdot \boldsymbol{l} I_{\nu}\right) \mathrm{d} \nu \mathrm{d} \Omega .
$$

Finally, substituting (34) into (33) gives the collision model for the electron gas related to radiative ionization, as

$$
\begin{aligned}
{\left[\frac{\delta\left(n_{\mathrm{e}} f^{\mathrm{e}}\right)}{\delta t}\right]_{\mathrm{P}}^{\mathrm{I}}=\frac{\boldsymbol{U}_{\mathrm{e}} f_{0 \mathrm{e}}^{\mathrm{e}}}{c k T_{\mathrm{e}}} \cdot \int K_{\nu} \boldsymbol{l}\left(I_{\nu}-I_{\nu 0}\right) \mathrm{d} \nu \mathrm{d} \Omega } & \\
& +\frac{\left(3 k T_{\mathrm{e}}-2 \varepsilon_{\mathrm{I}}-2 m_{\mathrm{e}} \boldsymbol{U}_{\mathrm{e}}{ }^{2}\right) R_{\mathrm{a}} \Phi_{0} f_{0 \mathrm{e}}^{\mathrm{e}}}{4\left(k T_{\mathrm{e}}\right)^{2} C_{\mathrm{v}}{ }^{\mathrm{I}}} \int K_{\nu}\left(I_{\nu 0}-I_{\nu}+\frac{1}{c} \overline{\boldsymbol{V}} \cdot \boldsymbol{l} I_{\nu}\right) \mathrm{d} \nu \mathrm{d} \Omega .
\end{aligned}
$$

Hence, Eqs. (36) - (38) complete the modelling of the radiation-initiated ionization term in model Eq. (10), and the model can be shown to obey Boltzmann H-theorem.

1 K. A. Brueckner and S. Jorna, Rev. Mod. Phys. 46, 325 [1974].

$2 \mathrm{H}$. Hora, in "Laser Interaction and Related Plasma Phenomena", edited by H. Schwarz and H. Hora, Plenum Press, New York 1, 383 [1970], and 2, 341 [1972].

3 J. W. Shearer and J. L. Eddleman, Phys. Fluids 16, 1753 [1973].

4 J. A. Stamper and D. A. Tidman, Phys. Fluids 16, 2024 [1973].

5 W. M. Manheimer and E. Ott, Phys. Fluids 17, 1413 [1974].

6 W. F. Phillips and V. S. Arpaci, J. Plasma Phys. 7, 235 [1972].
7 J. R. Saraf, Physica 70, 155 [1973] ; Phys. Rev. A 10, 214 [1974]. Z. Physik A 278, [1976], in press.

8 J. R. Saraf, J. Chem. Phys. 63, 2577 [1975].

9 J. R. Saraf, Phys. Fluids 19 [1976] ; Physica 83 c [1976], in press.

10 J. H. Clarke and C. Ferrari, Phys. Fluids 8, 2121 [1965].

11 D. G. M. Anderson, H. Baum, and M. Krook, in "Rarefied Gas Dynamics", edited by L. Trilling and H. Wachman, Academic Press, New York 1969, 1, 49.

12 W. F. Phillips and V. S. Arpaci, J. Plasma Phys. 13, 523 [1975].

13 D. H. Sampson, Radiative Contributions to Energy and Momentum Transport in a Gas, Interscience Publ., New York 1965, p. $32-34$. 